\title{
Terahertz Metamaterial Absorbers Implemented in CMOS Technology for Imaging Applications: Scaling to Large Format Focal Plane Arrays
}

\author{
Ivonne Escorcia Carranza, Member, IEEE, James P. Grant, John Gough, and David Cumming, Fellow, IEEE
}

\begin{abstract}
We present the design and fabrication of terahertz (THz) metamaterial (MM) absorbers and their monolithic integration into a commercial CMOS technology along with its respective readout electronics to produce a low-cost, uncooled, and high resolution THz camera. We first describe the work done on single band and broadband MM absorbers on custom substrates, then progress with a description of the integration of such resonators into a six metal layer $180 \mathrm{~nm}$ CMOS process and its coupling with two types of microbolometer sensors: Vanadium oxide (VOx) and silicon (Si) pn diode. Additionally, we demonstrate the integration of the $\mathrm{THz}$ sensors with readout electronics to form a monolithic $\mathbf{T H z}$ focal plane array (FPA). Reflection images of a metallic object hidden in a manila envelope are recorded using both the VOx and Si pn diode detectors, demonstrating the suitability of the technology for stand-off detection of concealed objects. Finally, we present the current work toward scaling this technology into a $64 \times 64$ FPA.
\end{abstract}

Index Terms-Uncooled bolometers, CMOS image sensors, terahertz imaging, terahertz metamaterials.

\section{INTRODUCTION}

$\mathbf{T}$ ERAHERTZ imaging has become a significant area of research owing to its unique characteristics. It is transparent to many non-conducting materials that are opaque in the visible and infrared (IR) bands. Moreover, on account of its low photon energy, it is non-ionizing and thus safe to biological tissue, in contrast to x-rays. Finally many materials, such as explosives and illicit drugs, have characteristic $\mathrm{THz}$ spectroscopic signatures that can be readily identified [1], [2]. Several applications such as stand-off security imaging of hidden objects and weapons [3], medical imaging for non-invasive diagnostics of skin cancer and dental care [4], [5], quality control methods for the food [6] and automotive industries [7] and non-contact imaging for conservation of paintings, manuscripts and artifacts [8] have derived from these unique characteristics. Despite the advantages offered by $\mathrm{THz}$ radiation, the widespread use of these

Manuscript received September 30, 2016; revised November 10, 2016; accepted November 14, 2016. This work is supported by the Engineering and Physical Sciences Research Council under Grants EP/I017461/1 and $\mathrm{EP} / \mathrm{J} 018678 / 1$.

I. Escorcia Carranza, J. P. Grant, and D. Cumming are with the Microsystems Technology Group, School of Engineering, University of Glasgow, Glasgow G12 8LT, U.K. (e-mail: Ivonne.EscorciaCarranza@glasgow.ac.uk; James.Grant@glasgow.ac.uk; David.Cumming.2@glasgow.ac.uk).

J. Gough is with the School of Engineering, University of Glasgow, Glasgow G12 8LT, U.K. (e-mail: John.Gough@glasgow.ac.uk).

Color versions of one or more of the figures in this paper are available online at http://ieeexplore.ieee.org.

Digital Object Identifier 10.1109/JSTQE.2016.2630307 applications still faces challenges due to the lack of suitable sources and detectors confining these applications to laboratory use. The primary focus is to overcome these issues by miniaturizing and lowering the production cost of $\mathrm{THz}$ imaging devices thus making them widely accessible.

Most $\mathrm{THz}$ images today are built up one pixel at a time, through raster scanning with single-point detectors. Such an approach, while producing high resolution images, has the drawback of extremely long acquisition times. Recent years have seen a drive to realize a focal plane array (FPA) of detectors with the goal of obtaining video rate $\mathrm{THz}$ imaging. There has been a strong desire to integrate $\mathrm{THz}$ detectors on a complementary metal oxide semiconductor (CMOS) platform in order to take advantage of the sophisticated electronic circuitry that is available for the biasing, read-out and addressing of large scale pixel arrays. Active sub-THz imaging systems have been demonstrated using a number of CMOS compatible detectors. An array of $4 \times 4$ Schottky barrier diode (SBD) pixels operating at $0.28 \mathrm{THz}$ with a minimum noise equivalent power (NEP) of $29 \mathrm{pW} / \sqrt{\mathrm{Hz}}$ was demonstrated in [9]. However operation at higher frequencies is desirable to provide better spatial resolution. According to Han et al., the SBDs efficiency is decreased 5 times from $0.28 \mathrm{THz}$ to $0.86 \mathrm{THz}$ due to the increment in bias current needed and the I-V curve deviating from the ideal characteristics; this in consequence increases the NEP. Al Hadi et al. presented a $32 \times$ 32 CMOS FPA using field effect transistors (FETs) operating at $0.856 \mathrm{THz}$ although the antenna was designed to operate from $0.70 \mathrm{THz}$ to $1.1 \mathrm{THz}$. This array is based on on-chip ring antennas coupled to NMOS detectors, it provides a maximum responsivity of $115 \mathrm{kV} / \mathrm{W}$ with a NEP of $12 \mathrm{nW}$ integrated over a $500 \mathrm{kHz}$ video bandwidth [10]. The disadvantage of this design is the increased pixel pitch due to the required antennas needed in each pixel and their performance reduction when operated above $1 \mathrm{THz}$ in array mode.

A number of uncooled $\mathrm{THz}$ detector arrays have been created using adaptations of longwave infrared (LWIR) quarter wavelength cavity structures to absorb the radiation coupled with microbolometer detectors. Microbolometers are thermal detectors composed of a material with a high temperature coefficient of resistance (TCR) that detect the incident radiation producing a temperature change [11]. This change is measured as an electrical signal such as a change in resistance or voltage. The most common microbolometer materials 
are Vanadium Oxide (VOx), amorphous silicon $(\alpha-\mathrm{Si})$ and Si pn diode [12].

Commercial quarter wavelength based FPAs have been reported in the literature e.g. an uncooled $320 \times 240$ FPA with an NEP of less than $100 \mathrm{pW}$ designed by NEC [13] and an uncooled $384 \times 384$ FPA with an NEP of 76.4 pW designed by INO [14]. CEA Leti has also realized a THz FPA implemented in a suspended bridge configuration. At $2.5 \mathrm{THz}$ the NEP is $32 \mathrm{pW}$ with a video rate of $25 \mathrm{~Hz}$ and a responsivity of 12.6 MV/W [15]. The major disadvantage of these types of detectors is their extensive and complex fabrication methods that significantly increase the production cost since the bolometer has to be positioned at a distance of $\lambda / 4$ from the substrate e.g. at $2.5 \mathrm{THz}(118.8 \mu \mathrm{m})$ this distance is $30 \mu \mathrm{m}$.

In order to have an efficient room-temperature $\mathrm{THz}$ detector it is necessary to have a suitable absorbing structure and a sensor. Due to the lack of existing natural THz absorbers, engineered metamaterials (MMs) have been employed as absorber elements. These artificially made materials have strong resonant absorption. In a MM absorber the radiation is absorbed in a device thickness of $<\lambda / 50$, overcoming the thickness limitation of traditional quarter wavelength devices. Both the absorption magnitude and frequency can be tailored by changing the MM structure geometry. Here we detail the novel integration of a THz MM absorber implemented in CMOS technology coupled to two different types of sensors: VOx and Si pn diode.

In this paper we present work on the development of high resolution, fully integrated, MM based THz FPAs. Section II presents the design of single band and broadband THz MM absorbers and their absorption characteristics. Their novel integration into a commercial six metal layer CMOS process coupled with two different types of detectors is described in Section III. The single pixel performance and imaging experiments of the VOx bolometer and the Si pn diode detectors are explained in Section IV. The last section details the steps taken to scale this technology into a high resolution system: a $64 \times 64$ FPA.

\section{DESIGN OF TERAHERTZ METAMATERIAL ABSORBERS}

The idea of artificial materials with both negative electric permittivity and magnetic permeability was studied by Veselago in 1968 [16]. He presented the possibility of creating lenses made out of left handed materials (LHMs) that would change the trajectory of the rays passing through them inversely to their normal path e.g. a convex lens would diverge and a concave lens would converge. This phenomenon was popularized by R.A. Shelby, D.R. Smith and S. Schultz in 2001 when they demonstrated experimentally the first artificial LHM. It was made of an array of combined split ring resonators (negative permeability) and metallic rods (negative permittivity) to produce a material with a negative refractive index [17]. This discovery led to the creation of engineered materials called MMs where the permittivity and permeability can be finely tuned to modify their electromagnetic (EM) properties. MMs consist of periodic arrays where both the shape and the unit cell are smaller than the resonance wavelength. They behave as frequency-selective surfaces when an incident EM wave interacts with them. The EM properties of these subwavelength arrays are modified by the size, shape and

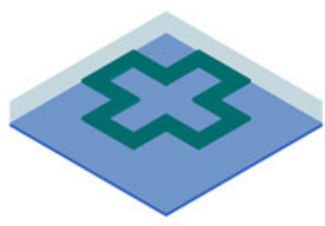

(a)

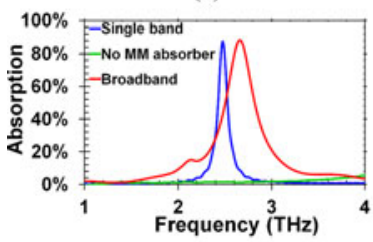

(c)

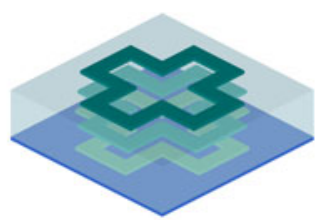

(b)

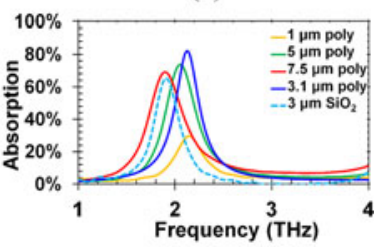

(d)
Fig. 1. Metamaterial Absorbers. (a) Single band MM absorber with a single cross-shaped ERR resonator and a ground plane separated by an insulator. (b) Broadband MM absorber with three cross-shaped ERR resonators, a ground plane and insulating layers separating the metallic layers. (c) Absorption characteristics of a single band (blue line) and broadband MM (red line). (d) Absorption characteristics of a single band MM with different insulator materials and thicknesses.

structure of the unit cell rather than the material composition [18]. MMs are usually composed of metallic electric ring resonators (ERRs) or split ring resonators (SRRs) and are able to absorb at any EM wave ranging from the microwave to visible wavelengths [19]. The effective permittivity and permeability can be independently controlled by pairing the resonators with metallic ground planes. This allows for the electric response to be tuned by changing the shape of the ERR or SRR, and the magnetic response by changing the spacing between the resonator and ground plane. Previous work has shown the design, fabrication and experimental characterization of custom $\mathrm{THz}$ MM absorbers focused on cross-shaped ERR structures for single band, dual band and broadband absorption [20]-[22]. Their performance will be described in this section.

\section{A. Single Band Absorbers}

Single band MM absorbers are composed of two metallic layers separated by a dielectric. The top metal layer contains an array of SRRs or ERRs while the bottom is a continuous ground plane. The absorption characteristics of this type of resonator are dependent on the interaction of the periodic subwavelength structures with the incident radiation resulting in absorption at one specific frequency. Single band MMs are narrow band by nature; typically their full width at half maximum (FWHM) is no more than $20 \%$ of the center frequency. Fig. 1(a) shows an example of a single band MM absorber with a cross-shaped resonator and a ground plane separated by an insulator. Its absorption characteristics are shown in Fig. 1(c) (blue line) demonstrating a maximum absorption magnitude of $87 \%$ at $2.47 \mathrm{THz}$.

\section{B. Broadband Absorbers}

Broadband MMs are composed of a stack of ERRs with varying dimensions separated by insulators. The resonant peaks depend on the number of ERRs in each unit cell. With judicious design, the individual resonances from each ERR result in peaks positioned close together that merge into one broad absorption spectrum. This type of absorber is characterized by their 
broad absorption in contrast to single band absorbers where the FWHM is increased to $48 \%$ for a broadband absorber compared to $20 \%$ for a single band absorber. For our research purposes, broadband MMs are used instead of single band to maximize the probability of strong absorption at the frequency of our $\mathrm{THz}$ source $(2.5 \mathrm{THz})$. This challenge is mainly present when integrating MMs in the metal-insulating layers of a CMOS process since the assumed values of the inter-metal dielectric refractive index used in simulation vary from the real values at $\mathrm{THz}$ frequencies.

An example of a broadband MM structure is shown in Fig. 1(b). It is composed of three stacked cross-shaped ERRs with different lengths and widths. Its absorption characteristics are shown in Fig. 1(c) (red line) and has a broad absorption band compared to the single band MM. This figure also shows that when there is no ERR structure, the absorption is minimal - the only loss occurs in the dielectric layer.

Previous research demonstrated that the frequency absorption peak of a cross-shaped resonator depends on the width and length of the ERR and the refractive index of the insulator, while the absorption magnitude is determined by the loss and thickness of the insulating layer. Fig. 1(d) shows the spectral response of a single band MM absorber with a polyimide dielectric thickness ranging from $1 \mu \mathrm{m}$ to $7.5 \mu \mathrm{m}$ compared to a $3 \mu \mathrm{m}$ thick $\mathrm{SiO}_{2}$ dielectric. The figure illustrates the increasing peak absorption magnitude in relation to thickness between $1 \mu \mathrm{m}$ and $3.1 \mu \mathrm{m}$, and a slight peak absorption value reduction at thicknesses greater than $3.1 \mu \mathrm{m}$. Additionally, the MM with a $3 \mu \mathrm{m}$ thick $\mathrm{SiO}_{2}$ dielectric shows a maximum absorption of $65 \%$ at $1.90 \mathrm{THz}[20]$.

\section{INTEGRATION OF THZ METAMATERIAL ABSORBERS INTO A COMMERCIAL CMOS PROCESS}

Miniaturizing and reducing the cost of the $\mathrm{THz}$ detector by monolithically integrating it into standard CMOS process would be a significant step towards the goal of a highly sensitive, low cost, uncooled and portable $\mathrm{THz}$ imaging system. The metalinsulator-metal structure of $\mathrm{THz} \mathrm{MM}$ absorbers lends itself to integration into the layers of a commercial CMOS process. This idea was implemented in a $180 \mathrm{~nm}$ six-metal layer process from Texas Instruments (CMOS9T5V). An example of the crosssection of this process is shown in Fig. 2(a). A broadband MM absorber with cross-shaped ERRs was integrated in the top three metal layers (M4 to M6) of the CMOS process. M3 was used as the continuous ground plane and M1-M2 were used as the interconnect for the readout electronics. The integration suffers from one challenge; the thickness of the oxide is process dependent therefore only the cross arm length and arm width can be modified to absorb at the required frequency.

The broadband MM absorbers were coupled with microbolometer sensors to have a complete $\mathrm{THz}$ detector. Two types of temperature sensing devices were paired with the $\mathrm{THz}$ MM absorber; VOx and Si pn diode. Both sensors are compatible with CMOS technology allowing for monolithic integration of the $\mathrm{THz} \mathrm{MM}$ absorber and electronics readout. To maximize the microbolometer response and increase the response speed it is necessary to minimize the thermal capacitance of the pixel,

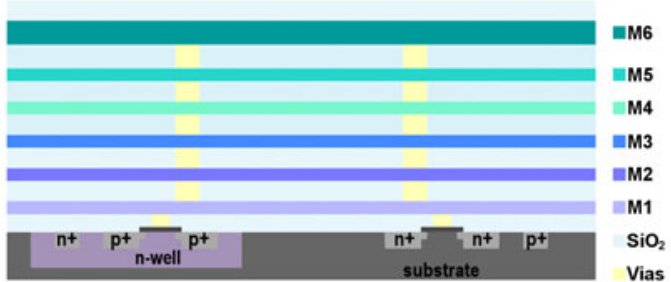

(a)

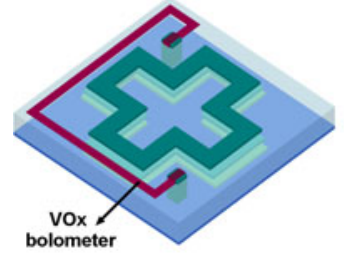

(b)

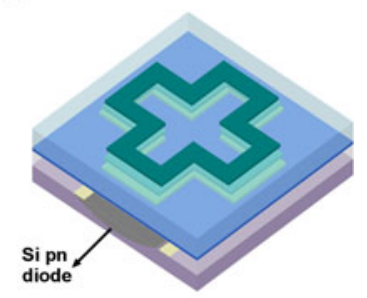

(c)
Fig. 2. 3D schematic of the MM based THz detectors integrated in a CMOS process. (a) Commercial six metal layer CMOS process. (b) Broadband MM absorber with a VOx sensor and (c) Si pn diode sensor integrated in the CMOS layers.

comprised of the THz MM absorber and VOx microbolometer, by reducing its thickness. The MM absorber, typically being a few microns thick, dominates the pixel performance. It is possible, by judicious design and selection of materials, to design a $\mathrm{THz} \mathrm{MM}$ absorber that can absorb $>70 \%$ of the incident radiation in a thickness of $\lambda / 50$ thereby maximizing device performance.

The VOx sensor was patterned in a series of post-processing steps above M6 and subsequently connected to the readout electronics using additional Ti/Au tracks. This resistive-type sensor was used due to its high TCR $(-2 \% / \mathrm{K}$ to $-3 \% / \mathrm{K})$ and relatively simple post-processing steps. Fig. 2(b) shows a 3D schematic of the broadband $\mathrm{THz}$ detector with three cross-shaped ERRs, continuous ground plane, insulator between metallic layers and patterned VOx in an "L" shape.

The Si pn diode sensor was investigated due to its existing integration in the CMOS process. In comparison to the VOx sensor, as it is already realized in the front-end of the CMOS process, no post-processing steps are required but its temperature coefficient of voltage $\left(\mathrm{TCV}_{\mathrm{D}}\right)$ is lower $(-2 \mathrm{mV} / \mathrm{K})$. In order to increase the $\mathrm{TCV}_{\mathrm{D}}$, three diodes in series were integrated in every pixel for a total theoretical $\mathrm{TCV}_{\mathrm{D}}$ of $-6 \mathrm{mV} / \mathrm{K}$. Fig. 2(c) shows a 3D schematic of the broadband $\mathrm{THz}$ detector with the Si pn diode integrated in the bottom layers of the CMOS process.

The broadband MM absorber was designed using 3D finite difference time domain (FDTD) simulations using Lumerical Inc. software. The simulation specifications are described in [23]. The absorber was designed to absorb at $2.5 \mathrm{THz}$ because of an existing atmospheric window at this frequency and because of the $\mathrm{THz}$ source available to us, $\mathrm{C} \mathrm{CO}_{2}$ pumped $\mathrm{CH}_{3} \mathrm{OH}$ (methanol) vapor laser, operating at $2.5 \mathrm{THz}$. The pixel size was $30 \mu \mathrm{m} \times 30 \mu \mathrm{m}$. Fig. 3(a) shows the refractive index of the materials composing the broadband MM absorber. The metallic layers from the three stacked ERRs are shown in red and the dielectric insulating layers are shown in light blue. 


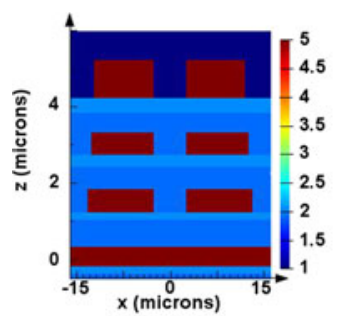

(a)

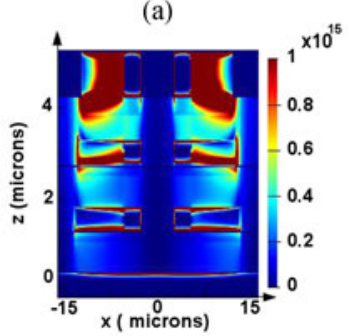

(c)

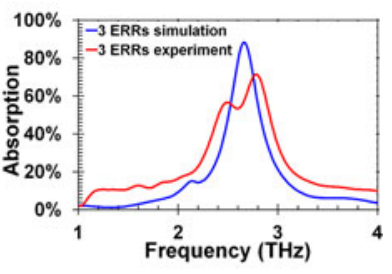

(b)

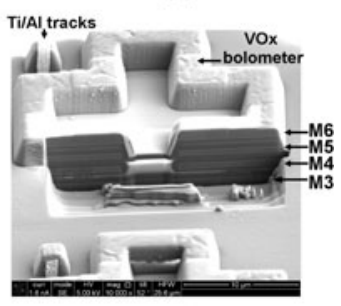

(d)
Fig. 3. Broadband MM absorber characteristics. (a) MM absorber crosssection showing the refractive index of the dielectric insulating layers (light blue) and metallic layers (red). (b) Simulation (blue line) and experimental (red line) spectral response of the MM absorber implemented in a CMOS9T5V process. (c) Simulated power distribution (per unit volume) in the $\mathrm{x}-\mathrm{z}$ plane at $y=$ $0 \mu \mathrm{m}$. (d) SEM picture showing a cross-section of the pixel.

The dielectric separating the metallic interconnects in the $0.18 \mu \mathrm{m}$ CMOS process is proprietary however it is nominally $\mathrm{SiO}_{2} . \mathrm{SiO}_{2}$ is an isotropic material therefore the refractive index is independent of the incident angle of the EM wave. Fig. 3(b) shows the simulated (blue line) and experimental (red line) spectral responses from the broadband absorber. The simulation shows a broad absorption peak of $50 \%$ magnitude at $2.5 \mathrm{THz}$. The devices were characterized under vacuum in a Bruker IFS 66v/S Fourier Transform Infrared Spectrometer. The experimental spectrum shows approximately $57 \%$ absorption at the frequency of interest. The difference between simulation and experimental results was attributed to the discrepancy between the real and assumed values of the inter-metal dielectric refractive index used in simulation. The actual values are not known at $\mathrm{THz}$ frequencies. Fig. 3(c) shows the simulated power distribution in the $\mathrm{x}-\mathrm{z}$ plane at $2.5 \mathrm{THz}$ demonstrating that the maximum absorbed radiation occurs in the dielectric between M5 and M6 ERRs. Fig. 3(d) shows an SEM picture of the crosssection of one pixel revealing the ground plane in M3, the three stacked ERRs in M4 to M6 separated by the dielectric, forming the MM absorber, and the subsequently post-processed addition of the VOx bolometer and Ti/Au connecting tracks. Note that the geometry of the VOx bolometer, a square of side $26 \mu \mathrm{m}$, is different from the "L" shape depicted in Fig. 2(b). Due to the topography of the as-received chip it proved impossible to reliably connect the square shaped bolometer to the M6 studs therefore the "L" shaped bolometer design, fabricated on a planar surface, was adopted.

\section{Single Pixel Performance Comparison Between VOX BOLOMETER AND SI PN DIODE}

The two types of THz detectors, VOx and Si pn diode, were characterized to determine the relevant figures of merit (FOM):

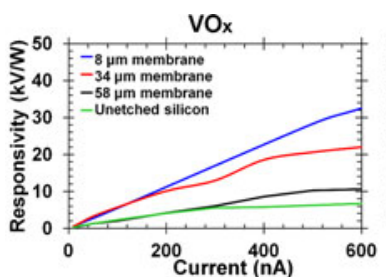

(a)

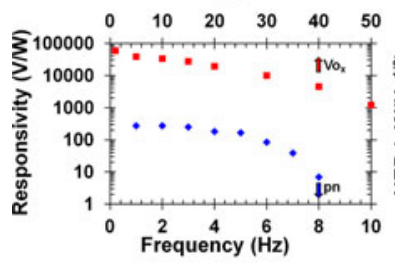

(c)

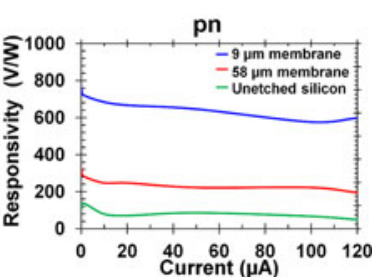

(b)

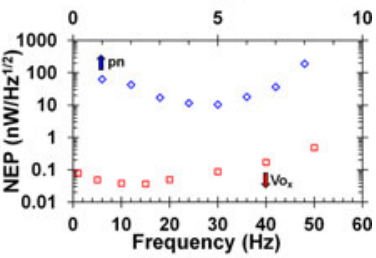

(d)

Fig. 4. Single pixel performance characteristics of the VOx and Si pn diode sensors. (a) VOx DC Responsivity for 4 membrane thicknesses showing the largest response of $32.65 \mathrm{kV} / \mathrm{W}$ from the thinnest membrane (blue line). (b) Si pn diode DC Responsivity showing the largest response of $684 \mathrm{~V} / \mathrm{W}$ from the thinnest membrane (blue line). Comparison of (c) responsivity as a function of frequency and (d) NEP for both sensors.

Responsivity $\left(R_{v}\right)$, TCR or TCV , NEP and thermal time constant $(\tau)$. Details of the characterization set ups and equipment used to measure each FOM can be found in [24], [25]. Here we present a comparison of the sensor's performance.

The thermal coefficient of both sensors was measured by placing the sensors in an environmental chamber and sweeping the temperature while measuring the change in output voltage when biased at different currents. The VOx sensor had an average TCR of $-2 \% / \mathrm{K}$ and the Si pn diode had a $\mathrm{TCV}_{\mathrm{D}}$ of $-5.4 \mathrm{mV} / \mathrm{K}$ at room temperature $\left(25^{\circ} \mathrm{C}\right)$.

The pixel's DC response, in terms of $\Delta \mathrm{V} / \mathrm{W}$, was measured from $10 \mathrm{nA}$ to $600 \mathrm{nA}$ and was established by measuring the area of the beam and incident power at the pixel. The focused beam had a diameter of $2 \mathrm{~mm}$ measured using a commercial FLIR camera. The incident power at the pixel was measured at the focus point and was calculated as $26 \mu \mathrm{W}$. The differential voltage $\left(\mathrm{V}_{\mathrm{ON} / \mathrm{OFF}}\right)$ was measured by monitoring the pixel's output voltage when the laser was on and off. The transimpedance configuration of the amplifiers converts the input bias current to voltage output having a gain equal to the microbolometer resistance. The output voltage has a linear relationship to the change in microbolometer resistance without signal amplification. In order to increase the responsivity, the thermal capacitance and conductance of the pixel was reduced by back etching the chip to remove the underlying silicon. Full details of the post-processing procedure can be found in reference [23]. In short, on-receiving the chips from the foundry, first the VOx bolometer was formed, then Ti/Au tracks were used to connect the bolometer to the M6 studs. Backside photolithography was used to pattern a circular window of radius $1 \mathrm{~mm}$ and the underlying silicon was removed using a standard inductively coupled plasma silicon etch process to make membranes with thicknesses ranging from $300 \mu \mathrm{m}$ (uneteched) to $8 \mu \mathrm{m}$. The responsivity was measured for four samples with different membrane thicknesses. Fig. 4(a) shows the DC responsivity of the four samples biased at different currents. The lowest responsivity is obtained from the 
unetched silicon (green line), owing to it having the thickest membrane $(308 \mu \mathrm{m})$ and therefore the largest thermal conductance. The largest responsivity of $32.65 \mathrm{kV} / \mathrm{W}$ was obtained with a $600 \mathrm{nA}$ bias current for the thinnest membrane $(8 \mu \mathrm{m}$ - blue line). For this sample, there was no silicon remaining, therefore there was no readout circuit. The second largest responsivity was $21.98 \mathrm{kV} / \mathrm{W}$ with a bias current of $600 \mathrm{nA}$ from the $34 \mu \mathrm{m}$ membrane (red line). This device had $26 \mu \mathrm{m}$ of silicon remaining allowing for integration of readout circuits in the pixel.

The $\mathrm{Si}$ pn diode DC response was measured from $10 \mathrm{nA}$ to $120 \mu \mathrm{A}$ for three membrane thicknesses to compare the increase in responsivity when reducing the volume of the pixel thus reducing the thermal capacitance and conductance. The results shown in Fig. 4(b) demonstrate the same pattern as the VOx sensors; the lowest responsivity comes from the unetched silicon (green line) and the largest responsivity of $684 \mathrm{~V} / \mathrm{W}$ from the thinnest membrane ( $9 \mu \mathrm{m}$ - blue line).

The responsivity as a function of frequency from the devices with the largest DC response was measured at different frequencies in the range of $1 \mathrm{~Hz}$ to $50 \mathrm{~Hz}$ for the VOx sensor and $1 \mathrm{~Hz}$ to $10 \mathrm{~Hz}$ for the Si pn diode. Fig. 4(c) shows the responsivity versus modulation frequency demonstrating the largest responsivity at $1 \mathrm{~Hz}$ for both sensors. For the VOx sensor (red squares), the responsivity at this frequency was $59 \mathrm{kV} / \mathrm{W}$ at a bias current of $2 \mu \mathrm{A}$ and for the Si pn diode (blue diamonds) the responsivity was $274 \mathrm{~V} / \mathrm{W}$ at a bias current of $10 \mu \mathrm{A}$. The thermal time constant was obtained from the responsivity versus frequency data and it was found to be $68 \mathrm{~ms}$ for the $8 \mu \mathrm{m}$ VOx sensor and $420 \mathrm{~ms}$ for the Si pn diode.

The root-mean-square $(\mathrm{rms})$ noise was measured to determine the NEP by placing the sensors in a Faraday cage and using a dynamic signal analyzer. The NEP was obtained by measuring the rms noise output voltage per square root unit bandwidth of the sensor divided by the responsivity modulated at different frequencies. Fig. 4(d) shows the NEP from the VOx sensor having a minimum NEP at $15 \mathrm{~Hz}$ of $108 \mathrm{pW} / \sqrt{\mathrm{Hz}}$ and a minimum $\mathrm{NEP}$ of $10.4 \mathrm{nW} / \sqrt{ } \mathrm{Hz}$ at $5 \mathrm{~Hz}$ for the $\mathrm{Si}$ pn diode.

In conclusion, the largest responsivity was obtained from the thinnest membranes due to the reduced thermal conductivity. Moreover, the thinner membrane devices had a shorter thermal time constant on account of the smaller thermal capacitance. The VOx sensor shows a better performance to the Si pn diode due to its superior temperature coefficient.

\section{A. Terahertz Imaging Experiments}

Imaging experiments were done with both the VOx and $\mathrm{Si}$ pn diode sensors in transmission and reflection mode using a gas laser operating at $2.5 \mathrm{THz}$. We present the results of the reflection mode imaging experiments only since this mode of operation is most usually required. The VOx and $\mathrm{Si}$ pn diode sensor with the largest responsivity were used to demonstrate their imaging capabilities. The experimental set up and object characteristics are described in detail in [25]. The experiment consisted in reflecting the focused $\mathrm{THz}$ beam from the surface of the aluminum cut out ' $\mathrm{T}$ ' shaped object, shown in Fig. 5(a), and

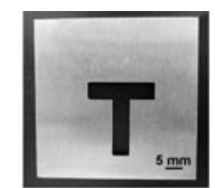

(a)

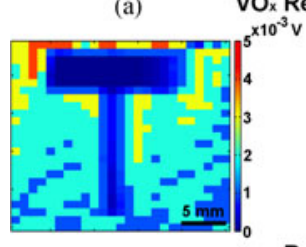

(c)

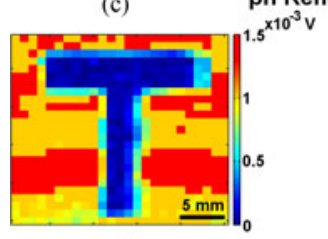

(e)

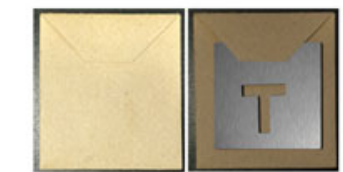

(b)

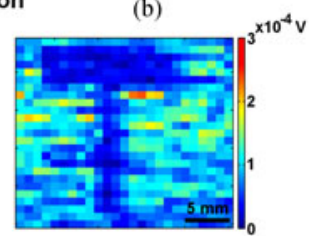

(d)

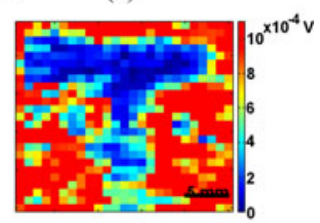

(f)
Fig. 5. Reflection mode Terahertz imaging using both detectors. (a) Optical image of the metallic object with a cut out "T" shape. (b) Metallic object hidden in a manila envelope. Reflection mode image of (c) the uncovered object and (d) covered object using the VOx detector. Reflection mode image of (e) the uncovered object and (f) covered object using the Si pn diode detector.

reflecting it back at an angle of $15^{\circ}$ for the VOx detector $(20 \mathrm{~cm}$ stand-off distance) and $25^{\circ}$ for the $\mathrm{Si}$ pn diode detector $(15 \mathrm{~cm}$ stand-off distance). The object was then hidden in a manila envelope, shown in Fig. 5(b), to demonstrate the capabilities of $\mathrm{THz}$ waves for identification of concealed objects. The images were formed by raster-scanning the single pixel detectors in the $x$-y direction in $1 \mathrm{~mm}$ steps to cover an area of $25 \mathrm{~mm} \times 25 \mathrm{~mm}$. The captured THz images obtained using the VOx sensor biased at $100 \mathrm{nA}$ for the uncovered object are shown in Fig. 5(c) and for the covered object in Fig. 5(d). The captured THz images obtained using the Si pn diode sensor biased at $10 \mu \mathrm{A}$ for the uncovered object are shown in Fig. 5e and for the covered object in Fig. 5f. The reflected signal non-uniformity surrounding the 'Ts' is due to the instability of the laser power and subsequently the detected power. The $\mathrm{THz}$ images demonstrate that both detectors can be effectively used in stand-off $\mathrm{THz}$ imaging applications.

\section{Scaling to a $64 \times 64$ Focal Plane Array}

A number of challenges existed in scaling the single pixel demonstration of a THz MM absorber implemented in CMOS to high resolution $64 \times 64$ pixel formats. A few of the notable challenges were: (1) devise a strategy to thermally isolate the pixels from each other to ensure no thermal cross-talk, (2) design the readout electronics such that the array could be read-out in real-time (50 frames per second) and (3) have the ability to select a bias current dependent on the VOx resistance in order not to saturate the output electronics. It is important to note that the single pixels described in Section IV were not connected to any electronics and the maximum voltage range we could measure using our source measure unit was $0 \mathrm{~V}$ to $20 \mathrm{~V}$ whereas the maximum voltage range available in the CMOS9T5V process is $0 \mathrm{~V}$ to $3.3 \mathrm{~V}$. Details of how we overcame these challenges and 


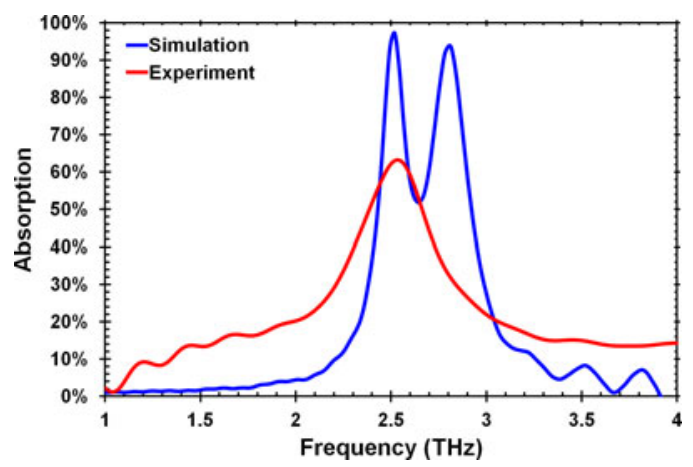

Fig. 6. Absorption spectra for the $64 \times 64$ FPA. Simulation (blue line) and experimental (red line) absorption spectra.

our approach to design a fully integrated $64 \times 64 \mathrm{THz}$ CMOS FPA are explained in this section.

\section{A. Pixel Design and Spectral Response}

The broadband MM absorber was modified from having three cross-shaped ERRs to two ERRs in M5 and M6. The dimensions of the ERRs were kept identical but the pixel size was increased to $40 \mu \mathrm{m} \times 40 \mu \mathrm{m}$ due to the requirements in post-processing which will be explained in Section (b). The ERR in M5 had an arm length of $23.5 \mu \mathrm{m}$, and an arm length of $22.5 \mu \mathrm{m}$ in M6 while both had a width of $10 \mu \mathrm{m}$. The ERR in M4 was substituted by a $26 \mu \mathrm{m} \times 26 \mu \mathrm{m}$ metal square, acting as the MM absorber ground layer. M3 was used as a sacrificial etch layer in a post-processing step to create free standing pixels above the integrated circuit (IC). Two $2.5 \mu \mathrm{m} \times 2.5 \mu \mathrm{m}$ posts in M1 to M6 were used to support each pixel and connect the VOx bolometer to the underlying electronics.

The simulated and experimental absorption characteristics of the $64 \times 64$ FPA were obtained following the same procedures described in Section III. Fig. 6 shows the comparison between the simulation (blue line) and experimental (red line) absorption spectra. In the simulation data the absorption spectra has two peaks, caused by the two stacked ERRs, at $2.5 \mathrm{THz}$ and $2.8 \mathrm{THz}$ with an absorption magnitude of $97 \%$ and $94 \%$ respectively. The experimental results show a broadband absorption peak centered at $2.5 \mathrm{THz}$ with an absorption magnitude of $62 \%$. These results confirmed greater than $60 \%$ absorption at the required frequency of $2.5 \mathrm{THz}$. The difference between simulation and experimental results may be due to the assumed values in simulation of the inter-metal dielectric refractive index, as mentioned previously in Section II and III, and the non-continuity in the ground plane allowing for some transmission.

\section{B. Post-Processing}

Before sending the chips to the foundry for fabrication, an overglass layer, typically only used to remove the silicon oxynitride passivation layer over the bond pads was drawn on top of the entire $5.9 \mathrm{~mm} \times 5.9 \mathrm{~mm}$ sensor array. This was done in order to be able to access the top metal, M6, studs that were to be subsequently connected to the VOx bolometer. On receiving the chips a series of post-processing steps were required: (1)

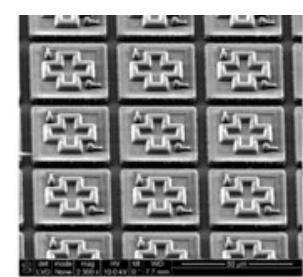

(a)

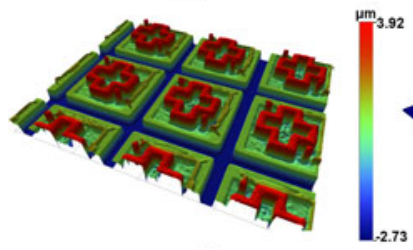

(c)

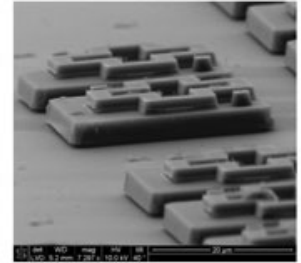

(b)

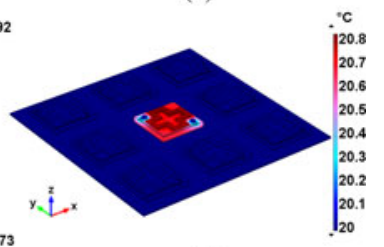

(d)
Fig. 7. Fabrication of THz MM based $64 \times 64$ FPA. (a) SEM picture showing a section of the array with free standing pixels. (b) SEM picture of scratched array revealing free standing and thus thermally isolated pixels. (c) Surface profiler image used to measure the vertical distance of the chip. (d) COMSOL simulations showing no cross-talk between pixels when they are thermally isolated.

definition of VOx bolometers, (2) connection of the bolometers to the underlying CMOS electronics and (3) thermal isolation of each pixel.

First, an electron-beam lithography registration process using a bi-layer of polymethylmethacrylate (PMMA) was used to define the bolometers and a reactive sputtering process $(5 \mu \mathrm{bar}$, $500 \mathrm{~W}$ RF power, 94\% Ar and 6\% $\mathrm{O}_{2}$ ), employed to deposit the VOx. The unwanted VOx was lifted-off by immersing the sample in a beaker of acetone. Next the bolometers were connected to the M6 studs using Ti/Au tracks by again using a PMMA bi-layer, electron beam evaporation of the metals and lift-off strategy. A $400 \mathrm{~nm} \mathrm{Si}_{3} \mathrm{~N}_{4}$ layer was then deposited via an inductively coupled plasma process on top of the CMOS chip to passivate the bolometers.

After formation of the bolometers and connection to the CMOS electronics the final, most challenging, step was thermal isolation of each pixel in the $64 \times 64$ array. This necessitated the removal of $400 \mathrm{~nm}$ of $\mathrm{Si}_{3} \mathrm{~N}_{4}, 3 \mu \mathrm{m}$ of fluorinated silica glass intermetal-dielectric (IMD5 to IMD3) and the $0.5 \mu \mathrm{m}$ thick sacrificial M3 Al layer while protecting the M6 Al ERR, VOx bolometer and Al bond pads. A $3 \mu \mathrm{m}$ wide opening was formed in a $7 \mu \mathrm{m}$ thick layer of SPR220-7 photoresist around the perimeter of each pixel and a cyclic $\mathrm{CHF}_{3} / \mathrm{O}_{2}$ reactive ion etch used to remove the $\mathrm{Si}_{3} \mathrm{~N}_{4}$ and IMD. Before wet etching the sacrificial M3 layer, sequential oxygen and argon cleans were performed to remove organic and fluorinated contaminants on top of the Al metal and ensure repeatable and uniform etching. A solution of $\mathrm{H}_{3} \mathrm{PO}_{4}: \mathrm{HNO}_{3}: \mathrm{CH}_{3} \mathrm{COOH}$ was used to remove the sacrificial Al layer. The sample was rinsed in $\mathrm{RO}$ water, transferred to a beaker of N-Methyl-2-pyrrolidone (NMP) at $80^{\circ} \mathrm{C}$ to remove the SPR220-7 photomask, rinsed again in RO water and hexamethyldisiloxane (HMDSO) then finally left to dry. The result, as shown in Fig. 7(a), was the THz MM absorber and VOx bolometers thermally isolated from the underlying CMOS IC. Fig. 7(b) shows an SEM picture of a scratched dummy chip used to determine that the M3 sacrificial Al layer was removed 


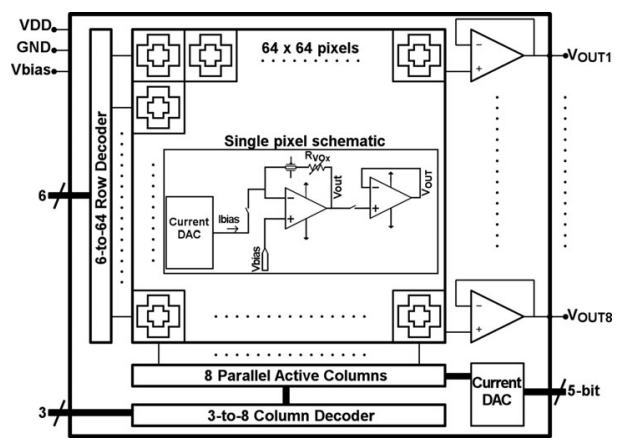

Fig. 8. Readout schematic of the $64 \times 64$ FPA and inset of single pixel readout schematic flow.

beneath the pixel and that the pixel was free standing. We also used surface profile interferometry (Bruker Contour) to determine the height of the bolometer to the integrated IC below and corroborate that the pixel was free standing and the thermal isolation was implemented successfully (see Fig. 7(c)). COMSOL simulations, shown in Fig. 7(d), were performed to verify that the pixel design resulted in no thermal cross-talk between adjacent pixels.

\section{C. $64 \times 64$ Parallel Readout}

The parallel readout architecture was based on the $5 \times 5$ array readout design explained in [25]. Such an architecture was implemented to increase readout speed. The array was designed using the same electronic components in the $5 \times 5$ array including the 5-bit DAC, 3 to 8 decoder, and amplifiers. In order to select 64 rows a 6 to 64 bit decoder with enable (EN) function was used. This decoder was built using nine 3 to 8 decoders where eight decoders provide 64 outputs. In order to enable the eight decoders, eight EN functions were needed. These EN functions were obtained from the ninth 3 to 8 decoder which controlled all the EN functions allowing for the proper selection of 64 rows. The row decoder needed a total of 6 bits provided externally.

The parallel readout process started with biasing the VOx microbolometers using a 5-bit current DAC. The external bias current selected depends on the resistance value of the microbolometer. For the chip that was tested, the average value was approximately $14 \mathrm{M} \Omega$. It was necessary to check that the output voltages were within the maximum voltage rails of the amplifiers $(0.3 \mathrm{~V}$ to $3.0 \mathrm{~V})$. After the current had been binary weighted with the external 5-bits of the digital input word, the readout process began by selecting eight columns at the same time and one row of each one of those columns. The result was that instead of having only one current DAC, the array has eight current DACs that bias eight columns at the same time. Therefore, the reading time is reduced by 8 times compared to the serial readout architecture. Each one of the 64 columns has an amplifier connected in a transimpedance feedback configuration to the microbolometer. Fig. 8 shows the parallel readout schematic of the $64 \times 64$ FPA. The inset in Fig. 8 shows the complete readout flow of one pixel, as the current $\left(\mathrm{I}_{\text {bias }}\right)$ biases the microbolometer $\left(\mathrm{R}_{\mathrm{VOx}}\right)$, the amplifier reads an output

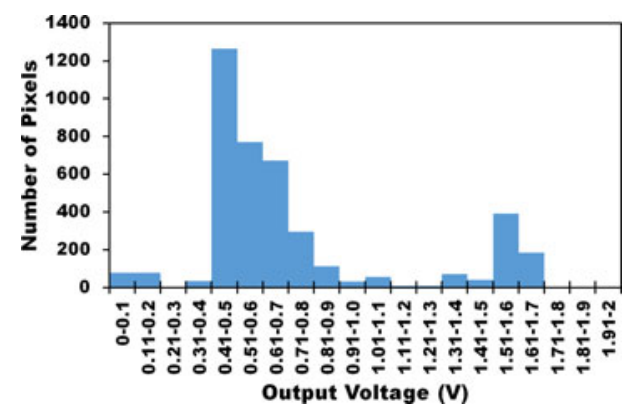

Fig. 9. Histogram plot of output voltages of the $64 \times 64$ FPA when biased at $50 \mathrm{nA}$.

voltage $\left(\mathrm{V}_{\text {out }}\right)$ between $0.3 \mathrm{~V}$ and $3.0 \mathrm{~V}$. In order to read the 8 columns simultaneously, each output from the amplifier is connected to a switch, activated by the 3 to 8 column decoder, and to one of the eight output amplifiers $\left(\mathrm{V}_{\text {OUT }}\right)$ used as buffers. Lastly, all the voltages are read out externally to create a voltage map.

\section{Results}

The post-processed chip was bonded to a ceramic pin grid array (CPGA) package (100 pins). Customized printed circuit boards (PCBs) were fabricated to bias and readout the electronic signals of the chip. The readout electronics were tested by current biasing the array and reading the voltage of each pixel in the $64 \times 64$ array. Fig. 9 shows a histogram plot of the 4096 pixels when biased at $50 \mathrm{nA}$. The data was taken using a Labview program. It can be seen from the histogram that $50 \%$ of the pixels have output voltages between $0.41 \mathrm{~V}$ to $0.7 \mathrm{~V}$ and that there is a degree of non-uniformity. Some of the reasons for the nonuniformity could be the damage caused by the post-processing steps, mismatch in the bias currents from the eight DACs, or mismatch in the deposition of the VOx films, resulting in different pixel resistances. Each one of the pixels can be compensated by employing off-chip image processing algorithms.

\section{CONCLUSION}

We have shown the progression from single band and broadband THz MM absorbers on bespoke custom substrates to their implementation in the metal and dielectric layers of a standard $180 \mathrm{~nm}$ CMOS process. We have coupled the CMOS THz MM absorbers with VOx and Si pn diode bolometers to form $\mathrm{THz}$ detectors and obtained minimum NEPs of $108 \mathrm{pW} / \sqrt{ } \mathrm{Hz}$ and $10.4 \mathrm{nW} / \sqrt{ } \mathrm{Hz}$ respectively. We have showcased the capability of both the VOx and Si pn diode devices for stand-off security imaging of concealed objects by forming reflection images of a metallic object with cut out ' $\mathrm{T}$ ' shape hidden in a manila envelope. We have successfully upscaled our proof-ofconcept single pixel $\mathrm{THz}$ detector to design, post-process and characterize a high resolution $64 \times 64 \mathrm{THz}$ CMOS FPA. With the ever improving power of $\mathrm{THz}$ sources and their increasing miniaturization, our MM based THz $64 \times 64 \mathrm{CMOS}$ technology is well suited for integration into $\mathrm{THz}$ imaging systems.

Moreover the beauty of MM structures is their scalability - they can operate from the millimeter wave to the visible. 
Incorporating THz MM absorbers with MWIR MM absorbers, as described in [26], and coupling to bolometers would render multi-wavelength FPA capable of forming co-axial images in two disparate wavebands. Hybridization of THz, MWIR and LWIR MM absorbers and visible plamonic colour filter structures, as described in [27], [28], has also been implemented potentially enabling co-axial visible, MWIR, LWIR and $\mathrm{THz}$ imaging. The hybridization of MM and plasmonic technologies in CMOS would result in a low-cost and compact multispectral camera.

\section{ACKNOWLEDGMENT}

The authors would like to thank the staff of the James Watt Nanofabrication Centre at the University of Glasgow for their contributions and Texas Instruments for provision of CMOS fabrication. The dataset associated with this research is available at [29].

\section{REFERENCES}

[1] X.-C. Zhang, "Terahertz wave imaging: Horizons and hurdles," Phys. Med. Biol., vol. 47, no. 21, pp. 3667-3677, 2002.

[2] K. Kawase, Y. Ogawa, Y. Watanabe, and H. Inoue, "Non-destructive terahertz imaging of illicit drugs using spectral fingerprints," Opt. Express, vol. 11 , no. 20, pp. 2549-2554, Oct. 2003.

[3] J. F. Federici et al., "THz imaging and sensing for security applicationsexplosives, weapons and drugs," Semicond. Sci. Technol., vol. 20, no. 7, pp. S266-S280, Jul. 2005.

[4] R. M. Woodward et al., "Terahertz pulse imaging in reflection geometry of human skin cancer and skin tissue," Phys. Med. Biol., vol. 47, pp. 3853-3863, 2002.

[5] A. Hall and J. M. Girkin, "A review of potential new diagnostic modalities for caries lesions," J. Dental Res., vol. 83, suppl. no. 1, pp. C89-C94, 2004.

[6] D. M. Mittleman et al., "Recent advances in terahertz imaging," Appl. Phys. B, Laser Opt., vol. 68, pp. 1085-1094, 1999.

[7] S. Krimi et al., "Highly accurate thickness measurement of multi-layered automotive paints using terahertz technology," Appl. Phys. Lett., vol. 109, no. 2, 2016, Art. no. 021105.

[8] K. Fukunaga, Y. Ogawa, S. Hayashi, and I. Hosako, "Terahertz spectroscopy for art conservation," IEICE Electron. Express, vol. 4, no. 8, pp. 258-263, 2007.

[9] R. Han et al., "Active terahertz imaging using schottky diodes in CMOS: Array and 860-ghz pixel," IEEE J. Solid-State Circuits, vol. 48, no. 10, pp. 2296-2308, Oct. 2013.

[10] R. Al Hadi et al., "A 1k-pixel video camera for 0.7-1.1 terahertz imaging applications in 65-nm CMOS," IEEE J. Solid-State Circuits, vol. 47, no. 12, pp. 2999-3012, Dec. 2012.

[11] D. S. Tezcan, S. Eminoglu, O. S. Akar, and T. Akin, "An uncooled microbolometer infrared focal plane array in standard CMOS," Proc. SPIE, Photodetectors, Mater. Devices VI, vol. 4288, no. 312, pp. 112-121, 2001.

[12] F. Niklaus, C. Vieider, and H. Jakobsen, "MEMS-based uncooled infrared bolometer arrays-A review," Proc. SPIE, MEMS/MOEMS Technol. Appl. III, vol. 6836, Nov. 2007, Art. no. 68360D.

[13] Uncooled THz Imager, NEC Corporation, Tokyo, Japan, pp. 1-2, 2014.

[14] C. Chevalier et al., "Introducing a $384 \times 288$ pixel terahertz camera core," Proc. SPIE, THz, RF, Millimeter, Submillimeter-Wave Technol. Appl. VI, vol. 8624, Mar. 2013, Art. no. 86240F.

[15] F. Simoens and J. Meilhan, "Terahertz real-time imaging uncooled array based on antenna- and cavity-coupled bolometers," Philos. Trans. A, Math Phys. Eng. Sci., vol. 372, no. 2012, pp. 1-12, 2014.

[16] V. G. Veselago, "The electrodynamics of substances with simultaneously negative values of $\epsilon$ and $\mu$," Sov. Phys. Uspekhi, vol. 10, no. 4, pp. 509-514, 1968.

[17] R. A. Shelby, D. R. Smith, and S. Schultz, "Experimental verification of a negative index of refraction," Science, vol. 292, no. 5514, pp. 77-79, 2001.

[18] A. Sihvola, "Metamaterials in electromagnetics," Metamaterials, vol. 1, no. 1, pp. 2-11, Mar. 2007.
[19] C. M. Watts, X. Liu, and W. J. Padilla, "Metamaterial electromagnetic wave absorbers," Adv. Opt. Mater, vol. 24, pp. OP98-OP181, Jun. 2012.

[20] J. Grant et al., "Polarization insensitive terahertz metamaterial absorber," Opt. Lett., vol. 36, no. 8, pp. 1524-1526, Apr. 2011.

[21] Y. Ma et al., "A terahertz polarization insensitive dual band metamaterial absorber," Opt. Lett., vol. 36, no. 6, pp. 945-947, Mar. 2011.

[22] J. Grant, Y. Ma, S. Saha, A. Khalid, and D. R. S. Cumming, "Polarization insensitive, broadband terahertz metamaterial absorber," Opt. Lett., vol. 36, no. 17, pp. 3476-3478, Sep. 2011.

[23] J. Grant et al., "A monolithic resonant terahertz sensor element comprising a metamaterial absorber and micro-bolometer," Laser Photon. Rev., vol. 7, no. 6, pp. 1043-1048, Nov. 2013.

[24] I. Escorcia, J. Grant, J. Gough, and D. R. S. Cumming, "Uncooled CMOS terahertz imager using a metamaterial absorber and pn diode," Opt. Lett., vol. 41, no. 14, pp. 3261-3264, 2016.

[25] I. E. Carranza, J. Grant, J. Gough, and D. R. S. Cumming, "Metamaterialbased terahertz imaging," IEEE Trans.THz Sci. Technol., vol. 5, no. 6, pp. 892-901, Nov. 2015.

[26] J. Grant, I. J. H. Mccrindle, C. Li, and D. R. S. Cumming, "Multispectral metamaterial absorber," Opt. Lett., vol. 39, no. 5, pp. 1227-1230, 2014.

[27] I. J. H. McCrindle, J. Grant, T. D. Drysdale, and D. R. S. Cumming, "Multi-spectral materials: Hybridisation of optical plasmonic filters and a terahertz metamaterial absorber," Adv. Opt. Mater, vol. 2, no. 2, pp. 149-153, 2014

[28] J. Grant, I. J. H. McCrindle, and D. R. S. Cumming, "Multi-spectral materials: hybridisation of optical plasmonic filters, a mid infrared metamaterial absorber and a terahertz metamaterial absorber," Opt. Express, vol. 24, no. 4, pp. 3451-3463, Feb. 2016.

[29] 2016. (Online). Available: http://dx.doi.org/10.5525/gla.researchdata.354

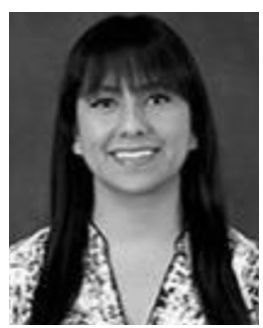

Ivonne Escorcia Carranza (M '08) received the B.E. degree from John Brown University, Siloam Springs, AR, USA, in 2007, the M.S.E.E. degree from the University of Arkansas, Fayetteville, AR, USA, in 2010, and the Ph.D. degree in electronics and electrical engineering from the University of Glasgow, Glasgow, U.K., in 2015. She is currently a Research Assistant in the School of Engineering, University of Glasgow, working with the Microsystem Technology group. She is the Lead Researcher in the design and characterization of $\mathrm{CMOS} \mathrm{THz}$ detectors. Her research interests include mixed-signal IC design, CMOS sensors and $\mathrm{THz}$ imaging. She is a member of the Eta Kappa Nu.

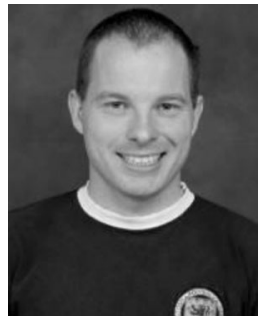

James P. Grant received the B.Sc. and Ph.D. degrees in physics from the University of Glasgow, Glasgow, U.K, in 2002 and 2006, respectively.

He is currently a Postdoctoral Research Fellow in the School of Engineering, University of Glasgow, where his research interests include nanofabrication, metamaterial devices; plasmonics, terahertz systems, sensors and imaging and CMOS electronics.

John Gough is currently with the UESTC joint engineering program at the University of Glasgow, Glasgow, U.K. He has previously worked as a Design Center Manager at Texas Instruments, Greenock, U.K., the Design Manager for National Semiconductor and Telecoms Division. He has worked on products, such as subscriber line interface devices and $\mathrm{S}$ - interface devices for ISDN products. He holds patents on a switched capacitor dual lamda oxygen sensor interface circuit and low dropout regulators. The later device was used in major cell phone platforms and sold close to two billion units worldwide.

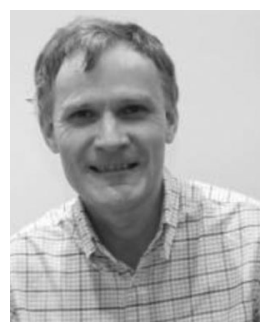

David Cumming (F'13) holds the B. Eng. degree in electronic and electrical engineering (Glasgow, 1989) and the $\mathrm{Ph} . \mathrm{D}$. degree in microelectronics (Cambridge 1993). He is currently the Head of Engineering at the University of Glasgow, Glasgow, U.K. His research interests include CMOS ion sensitive array technology has been commercialized into the Ion Torrent next generation sequencing system. He is a Royal Society Wolfson Merit Award Holder. 